\title{
Manuscrítica, Revista de crítica genética, n 17, 2009
}

\section{Maria Ignez Mena Barreto}

\section{OpenEdition}

\section{Journals}

Édition électronique

URL : https://journals.openedition.org/genesis/568

DOI : 10.4000/genesis.568

ISSN : 2268-1590

\section{Éditeur :}

Presses universitaires de Paris Sorbonne (PUPS), Société internationale de génétique artistique littéraire et scientifique (SIGALES)

\section{Édition imprimée}

Date de publication : 1 janvier 2011

Pagination : 229-230

ISBN : 978-2-84050-749-9

ISSN : 1167-5101

Référence électronique

Maria Ignez Mena Barreto, «Manuscrítica, Revista de crítica genética, n 17, 2009 », Genesis [En ligne], 32 | 2011, mis en ligne le 17 juin 2013, consulté le 11 janvier 2023. URL : http:// journals.openedition.org/genesis/568; DOI : https://doi.org/10.4000/genesis.568 


\section{Comptes rendus d'ouvrages}

Manuscrítica, Revista de crítica genética, $n^{\circ}$ 17, Editora Humanitas, 2009.

\section{Compte rendu par Maria Ignez Mena Barreto}

Le lecteur de Genesis qui suit de près l'évolution de la critique génétique au Brésil est peut-être déjà familiarisé avec la nouvelle formule de la revue Manuscrítica, dont ce numéro 17 constitue la troisième livraison. Créée en 1990 par 1'APML (Associação dos Pesquisadores do Manuscrito Literário, rebaptisée depuis 2006 APCG Associação dos Pesquisadores em Crítica Genética), la revue Manuscrítica ${ }^{1}$ a quitté la présentation dépouillée de la publication universitaire confidentielle qu'elle avait longtemps gardée pour un nouveau format plus en accord avec sa réelle diffusion et son statut de principal organe de publication de la recherche en génétique textuelle d'Amérique latine. Outre une maquette élégante, un plus beau papier, une mise en page soignée, une typographie plus aérée et plus agréable à lire, la nouvelle formule a été restructurée en six sections : Passando à limpo, dédié à l'agenda de l'APCG ; Fac-símile, avec, dans ce numéro, la reproduction d'une page manuscrite de Benveniste ; Atelié, regroupant les contributions de quatre chercheurs chevronnés : Celia Nunes Silva et Silvia Maria Guerre Anastácio, qui signent ensemble une contribution à la théorie dans «Uma visão sistémica do processo criador » (Une vision systémique du processus de création); Kleber Pereira dos Santos qui, dans «A gestação d' $O$ ventre » (La gestation d'O ventre) se penche sur un bel exemple de genèse de l'imprimé et Juan Eduardo Bonin, qui, dans « Génesis política del discurso religioso : Pueblo y Populismo en Iglesia y comunidad nacional (1981) » (Genèse politique du discours religieux : peuple et populisme dans Église et communauté nationale [1981]) aborde la question de la genèse d'une écriture collective. La revue comporte encore une quatrième section intitulée Incipit, où l'on retrouve un article consacré à Proust, " "La fenêtre éclairée" : uma escrita constelar » (« La fenêtre éclairée » : une écriture en constellation) signé par Carla Cavalcanti e Silva ; une section de comptes rendus intitulée Comentário ; une section Traduction, où l'on peut lire en portugais l'article «Conceptualisation et textualisation dans le manuscrit de l'article "Le langage et l'expérience humaine" d'Émile Benveniste. Une contribution à la génétique de l'écriture en sciences humaines ", d'Irène Fénoglio ${ }^{2}$ et une dernière section, Diálogo, consacrée dans ce numéro à un entretien accordé par l'écrivain brésilien Pedro Nava à Edina Regina P. Panichi.

Dans «A gestação d' $O$ ventre », Kleber Pereira dos Santos tente de déceler la présence de l'intertexte existentialiste dans la production du premier roman d'Heitor Cony, $O$ ventre, à travers l'étude comparative d'un ensemble de documents dont la rédaction s'étale sur une quarantaine d'années : une version dactylographiée datée de 1955 appartenant manifestement à une phase assez avancée de la genèse, l'édition originale de 1958 et deux éditions postérieures ayant fait l'objet d'importants remaniements, la sixième, datée de 1987, et la huitième et dernière édition revue par l'auteur, datée de 1998.
Dans le cadre de cet article, l'auteur porte son attention tout d'abord sur les transformations du paratexte : première et quatrième de couverture, rabats, titres, dédicaces et épigraphes, puis sur le remaniement de la structure d'ensemble du roman et les modifications des titres de chapitre qu'il entraîne successivement. Dans le sillage des propositions théoriques avancées par Roberto Zular et Claudia Amigo Pino dans leur essai Escrever sobre escrever : uma introdução à critica genética ${ }^{3}$, la visée est ici moins celle d'effectuer un recensement exhaustif de toutes les modifications apportées au texte que d'y repérer un mouvement d'écriture orienté par une problématique de recherche spécifique. Il ressort de l'analyse proposée par Kleber Pereira dos Santos que le progressif effacement du discours auctorial et des marques de subjectivité dans le paratexte, tout autant que le remaniement de la structure d'ensemble, répondent à la recherche d'une plus grande cohérence formelle entre le roman et l'arrière-fond philosophique qui guide obliquement sa rédaction.

La contribution de Juan Eduardo Bonin, « Génesis política del discurso religioso : Pueblo y Populismo en Iglesia y comunidad nacional (1981) », analyse les variations dans l'emploi du terme

1. Voir le compte rendu qu'en donna Régis Salado des deux premiers numéros dans Genesis, n 3, 1993, p. 165-171.

2. Paru en français dans Modèles linguistiques, vol. XXX, n 59, « Génétique de la production écrite et linguistique », volume coordonné par Irène Fenoglio et Jean-Michel Adam, 2009, p. 71-99.

3. São Paulo, WMF Martins Fontes, 2008. 
« peuple » à travers le processus de rédaction du document «Église et communauté nationale » (1981), l'un des documents de l'épiscopat argentin les plus influents sur un plan politique, publié dans les dernières années de la dictature militaire. La rédaction de ce document a été confiée à un groupe d'acteurs appartenant au Movimento de Sacerdotes para el Tercer Mundo (MSTM) (Mouvement des prêtres pour le tiers-monde), mouvement qui a surgi et s'est développé en Argentine entre 1968 et 1973, qui a cherché à concilier le concile Vatican II et l'action politique sur un plan à la fois théologique et pastoral. L'analyse de ce dossier, composé de quatre brouillons dactylographiés portant des corrections autographes, permet à Juan Eduardo Bonin de mesurer l'effort que déploient les rédacteurs pour traduire les contenus idéologiques du secteur du catholicisme qu'ils incarnent dans les termes plus généraux du dispositif discursif de la Conférence épiscopale argentine (CEA). La comparaison entre le dernier dactylogramme et le texte publié montre néanmoins à quel point les évêques ont surveillé cette opération.

Dans une approche plus classique en génétique textuelle, "La fenêtre éclairée" : uma escrita constelar » de Carla Cavalcanti e Silva tente de saisir la dynamique compositionnelle à l'œuvre dans les brouillons de Proust à travers l'analyse du mouvement d'écriture qui, d'une première ébauche conduit à la rédaction définitive de cet épisode bien connu de $L a$ Prisonnière. En suivant la progression de ce mouvement à travers l'étude des cahiers 53 et 55, notamment, Carla Cavalcanti e Silva souligne d'abord l'absence de linéarité dans l'usage que Proust fait du support cahier : utilisation discontinue des rectos, puis des versos, avec aménagement de blancs remployés lors d'étapes ultérieures, détachement et déplacement des feuillets, témoignant d'un mouvement d'aller et retour incessant entre les deux cahiers. À cet usage non linéaire du support répond une écriture qui, elle aussi, progresse de façon discon- tinue : concentration puis éclatement des contenus ou signifiés thématiques, déplacement et dissémination des motifs, traitement en parallèle des connotations euphorique ou dysphorique qui leur sont associées, puis fusion ou tressage dans la construction d'ambivalences, bref, tout un ensemble complexe d'opérations que Carla Cavalcanti e Silva porte à la lumière et qui la conduisent à parler de l'écriture proustienne comme d'une écriture en constellation.

« Uma pequena esfera iridescente » (Une petite sphère iridescente) est un hommage très personnel que rend Claudia Amigo Pino à son maître et ambassadeur de la critique génétique au Brésil, le Prof. Philippe Willemart. Présenté lors du lancement du livre Processos de criação 4 (Processus de création), ce texte est l'occasion pour Claudia Amigo Pino de retracer sa rencontre avec une pensée qui se poursuit inlassablement à contre-courant de la doxa universitaire et qui, animée par une grande ambition universaliste, opère par coups de sonde portés sur des champs aussi éloignés que la physique et la psychanalyse, la biologie et la philosophie, en passant par les mathématiques et la théorie littéraire pour s'affronter aux questions que suscite l'approche des manuscrits d'écrivain : qu'est-ce qu'écrire? comment écrit-on? en quoi les processus d'écriture nous éclairent-ils sur la manière qu'a l'humain de penser et d'ordonner le monde qui l'entoure ? Le commentaire va au-delà du compte rendu d'ouvrage classique pour constituer une invitation enthousiaste à la découverte de l'ensemble de la (très abondante) production de Philippe Willemart.

Le numéro se clôt sur une visite en éclaireur de l'atelier de Pedro Nava par Edina Regina P. Panichi dont se réjouiront sûrement les généticiens souhaitant se lancer dans l'étude des manuscrits de cet écrivain décédé en 1984, à une époque où la critique génétique ébauchait à peine ses premiers pas au Brésil.
Julie LeBlanc, Genèses de soi. L'écriture du sujet féminin dans quelques journaux d'écrivaines, Montréal, Éditions du remue-ménage, 2008, 238 p.

\section{Compte rendu par Catherine Viollet}

Genèses de soi, de Julie LeBlanc (professeur à l'université de Toronto, Canada), relève, de manière stimulante et novatrice, un double défi : appliquer la génétique textuelle à l'écriture diaristique - démarche qui peut sembler paradoxale au premier abord ; et l'appliquer à un corpus d'auteurs francophones de sexe féminin - fait suffisamment exceptionnel, dans la critique génétique de langue française ${ }^{5}$, pour qu'il mérite d'être souligné.

Si le titre choisi évoque l'entreprise, commune aux différents auteurs, d'autoengendrement, de construction du sujet dans et par l'écriture, les cas étudiés abordent des configurations fort diverses : journal de voyage, journal de genèse, journal palimpseste, roman-journal... S'ils présentent des écarts par rapport au genre canonique, tous possèdent une dimension génétique - des phases de réécriture et de métamorphose ; ils ont aussi en commun des préoccupations esthétiques, et le souci d'exprimer un point de vue féminin, voire féministe.

Une première partie, intitulée « Genèse d'un journal », réunit deux études qui ont pour même objet la transformation d'un journal dont une version a été diffusée oralement sur Radio-Canada, puis remaniée pour être publiée.

Journal intime, de Madeleine Ouellette-Michalska, radiodiffusé à partir de l'été 1982, se compose de nombreux cahiers (déposés à la Bibliothèque natio-

4. São Paulo, Perspectiva, 2009.

5. Il suffit, pour s'en convaincre, de feuilleter la «Bibliographie : études génétiques, éditions, manuscrits » de l'un des derniers numéros de la revue Genesis, recensant les publications dans ce domaine de janvier 2008 à janvier 2010 : moins de $4 \%$ des publications citées concernent des auteurs de sexe féminin. Voir Genesis, $n^{\circ} 30$, « Théorie : état des lieux », p. 285-295. 